\title{
Three-dimensional speckle tracking echocardiography in evaluating left ventricular function in patients with triple vessel coronary artery disease
}

Xiuxiu Cui ${ }^{1}$, Huaying Bo ${ }^{1}$, Yu Dong ${ }^{1}$, Ying Wang ${ }^{1}$, wenxing chang ${ }^{2}$, ying li $^{3}$, Tingting $\mathrm{Yu}^{1}$, and Guangsen $\mathrm{Li}^{1}$

${ }^{1}$ Affiliation not available

${ }^{2}$ Second Affiliated Hospital of Dalian Medical University

${ }^{3}$ Second affiliated Hospital of Dalian Medical University

February 28, 2021

\begin{abstract}
AIM Using three-dimensional ultrasound speckle tracking echocardiography (3D-STE) to evaluate left ventricular (LV) function in patients with triple vessel coronary artery disease(TVD) without myocardial infarction. METHODS Sixty patients with TVD without myocardial infarction were divided into two groups according to the results of coronary angiography. Group B $(\mathrm{n}=31): 50 \%[?]$ the stenosis rate of all triple vessel coronary arteryi75\%; Group $\mathrm{C}(\mathrm{n}=29)$ :the stenosis rate of all triple vessel coronary artery[?]75\%. Thirty healthy subjects were recruited as the group A. We measured LV end-diastolic and end-systolic volume (LVEDV, LVESV) and LV ejection fraction (LVEF) using real-time dynamic three-dimensional echocardiography. The 3D-STE parameters of LV included global longitudinal strain (GLS), global area strain (GAS), global radial strain (GRS) and global circumferential strain (GCS). The correlation between 3D-STE parameters and NT-proBNP were analyzed by Pearson

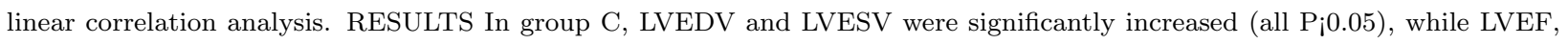

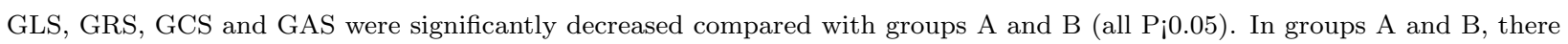
were no statistical differences in LVEDV, LVESV and LVEF. However, GLS, GCS and GAS were lower in group B than in group A (all $\mathrm{P} ; 0.05)$. The correlation analysis showed a negative correlation between the absolute values of GLS, GRS, GCS, GAS and NT-proBNP in group $\mathrm{C}(\mathrm{r}=-0.866 、-0.587 、-0.428 、-0.600, \mathrm{P} j 0.001 、 \mathrm{P}=0.001 、 \mathrm{P}=0.020 、 \mathrm{P}=0.010)$. CONCLUSIONS Our study shows that 3D-STE can evaluate the LV function in patients with triple vessel coronary artery disease without myocardial infarction through multiple strain parameters.
\end{abstract}

Three-dimensional speckle tracking echocardiography in evaluating left ventricular function in patients with triple vessel coronary artery disease

CUI Xiu-xiu, DONG Yu, WANG Ying, CHANG Wen-xing, LI Ying, YU Ting-ting, LI Guang-sen*

Department of Ultrasound, The Second Affiliated Hospital of Dalian Medical University,

Dalian, Liaoning 116027, China

Abstract: AIM Using three-dimensional ultrasound speckle tracking echocardiography (3D-STE) to evaluate left ventricular (LV) function in patients with triple vessel coronary artery disease(TVD) without myocardial infarction. METHODS Sixty patients with TVD without myocardial infarction were divided into two groups according to the results of coronary angiography. Group B ( $\mathrm{n}=31): 50 \%[?]$ the stenosis rate of all triple vessel coronary artery;75\%; Group C $(\mathrm{n}=29)$ :the stenosis rate of all triple vessel coronary artery[?]75\%. Thirty healthy subjects were recruited as the group A. We measured LV end-diastolic and end-systolic volume 
(LVEDV, LVESV) and LV ejection fraction (LVEF) using real-time dynamic three-dimensional echocardiography. The 3D-STE parameters of LV included global longitudinal strain (GLS), global area strain (GAS), global radial strain (GRS) and global circumferential strain (GCS). The correlation between 3D-STE parameters and NT-proBNP were analyzed byPearson linear correlation analysis. RESULTS In group C, LVEDV and LVESV were significantly increased (all $P$ ¡0.05), while LVEF, GLS, GRS, GCS and GAS were significantly decreased compared with groups $\mathrm{A}$ and $\mathrm{B}$ (all $P ; 0.05$ ). In groups $\mathrm{A}$ and $\mathrm{B}$, there were no statistical differences in LVEDV, LVESV and LVEF. However, GLS, GCS and GAS were lower in group $\mathrm{B}$ than in group A (all $P$ i0.05). The correlation analysis showed a negative correlation between the absolute values of GLS, GRS, GCS, GAS and NT-proBNP in group C $(r=-0.866 、-0.587 、-0.428 、-0.600, P$ ¡0.001 $P=0.001 、 P=0.020 、 P=0.010)$.CONCLUSIONS Our study shows that $3 \mathrm{D}-\mathrm{STE}$ can evaluate the LV function in patients with triple vessel coronary artery disease without myocardial infarction through multiple strain parameters.

Key words: Three-dimensional speckle tracking echocardiography, Coronary artery disease, triple vessel coronary artery disease,Left ventricular function, NT-proBNP

\section{Introduction}

Coronary heart disease (CHD) has been a hot spot in cardiovascular disease research. It is a heart disease caused by of the formation of coronary atherosclerotic plaque, which result in stenosis or obstruction of the vascular lumen, leading to myocardial ischemia, hypoxia and necrosis. According to the report published in 2016, CHD accounts for $20 \%$ of deaths in Europe, and is still one of the diseases with the highest morbidity and mortality in the world ${ }^{[1,2]}$. However, CHD involving three vessels disease (TVD) is a more extensive, diffuse and more serious disease than common CHD, and the left ventricular (LV) function can be impaired earlier. The main function of the LV is to transport blood to all parts of the body and undertake the blood supply to the whole body. So the effective evaluation of LV function in patients with TVD is very meaningful to the treatment and prognosis of patients. Traditional echocardiography has become the first choice for clinicians to diagnose heart disease, but it is less sensitive in early identification of cardiac function changes ${ }^{[3]}$. The 3D-STE is a quantitative tool to evaluate LV function with high accuracy and reliability ${ }^{[4]}$. Nesser et al. ${ }^{[5]}$ found that 3D-STE in assessment of LV systolic function had a remarkable correlation with cardiac MRI. At present, 3D-STE has been widely applied to disease diagnosis and academic research in terms of cardiac ${ }^{[6-8]}$. This study used 3D-STE to quantitatively analyze the changes of systolic LV strain parameters and evaluate LV function in patients with TVD without myocardial infarction.

\section{Methods}

Study population and grouping principles

Our study enrolled patients, who had been clinically diagnosed with CHD at the department of cardiology, from hospital admissions made between June 2019 and December 2020 in our hospital. Our diagnostic criteria for CHD was consistent with the diagnostic and therapeutic guidelines of CHD from the European Society of Cardiology (ESC) published in $2013^{[9]}$. Among them, sixty patients with TVD were selected as the experimental group. Diagnostic criteria of TVD was as follows: coronary angiography showed that the diameter stenosis rates of three subepicardial vessels (left anterior descending coronary artery, circumflex coronary artery, right coronary artery) and / or their main branches were all [?] 50\%. Sixty patients with TVD were divided into two groups: 31 patients with 50\%[?] the stenosis rate of all triple vessel coronary artery; $75 \%$ served as group B (age, $45^{\sim} 77$ years; mean age, $57.41 \pm 8.29$ years; ratio of females to males: 20:11), 29 patients with all three-vessel stenosis rate[?] $75 \%$ served as group C (age, 44 71 years; mean age, 57.37+-8.16 years; ratio of females to males: 20:9). At the same time, we recruited 30 gender and agematched healthy subjects (age, 41 69 years; mean age, 55.43+-7.54 years; ratio of females to males: 18:12) as group A. And all of the above participants were in sinus rhythm.

Exclusion criteria included: 1) therapeutic history of coronary revascularization; 2) myocardial infarction; 3) cardiac injury caused by coronary heart disease-related complications, such as papillary muscle rupture, ventricular wall rupture, interventricular septal perforation; 4) cardiac enlargement and cardiac dysfunction 
resulted by other types of heart disease, such as dilated cardiomyopathy, congenital heart disease, rheumatic heart disease, chronic pulmonary heart disease, valvular disease; 5) combined with hypertension, diabetes, nephrotic syndrome that contribute to heart damage. We also excluded the poor quality images.

Before the examination, we recorded the basic information of all participants on the checklist including name, gender, age, height and weight, then calculated body mass index (BMI, $\mathrm{kg} / \mathrm{m} 2$ ). After taking a 10 min break, a physician who was blind the purpose of the study measured the blood pressure (BP) and the heart rate (HR) of all participants. And $4 \mathrm{~mL}$ venous blood samples were retrieved in fasting in the morning from three groups of participants, and the serum was obtained by centrifuging venous blood at $3000 \mathrm{r} / \mathrm{min}$ for $10 \mathrm{~min}$ and stored in the refrigerator(-30). Then the content of N-terminal pro-brain natriuretic peptide (NT-proBNP) of the serum were measure by photochemical method.

The present study was accomplished with the consent of the Ethics Committee of our hospital. All participants fully understood the potential risks and objective of this study, and signed the informed consents before all examinations were carried out.

Conventional echocardiographic measurements

In our study, a GE Vivid E9 doppler ultrasound instrument equipped with an M5S-D probe (1.5-4.5 MHz) was introduced to obtain 2-dimensional images. The ultrasound examination was conducted when the subjects lay in the left lateral decubitus position and keep steady breathing with connecting a three-lead electrocardiography (ECG). Three groups of participants completed an assessment of echocardiography from multiple views including the LV long-axis, apical 4-chamber and apical 2-chamber. We measured the conventional parameters which included interventricular septal thickness and LV posterior wall thickness at end-diastolic (IVSTd and PWTd), LV end-diastolic diameter (LVDd) and LV end-systolic diameter (LVDs), transmitral peak early and late inflow diastolic velocity ( $\mathrm{E}$ and $\mathrm{A}$ ), early-diastolic tissue velocity of septal and lateral mitral annulus (Sept e' and Lat e'), then computed respectively E/A and E/mean e'. We used the views of apical 2-chamber and 4-chamber to measure the left atrial maximum volume at the LV end-systole by modified Simpson's rule, then calculated left atrial maximum volume index (LAVImax).

Real-time three-dimensional echocardiography and 3D-STE measurements

To acquire 3D echocardiographic LV full volume images, we used a matrix-array 4V-D probe (1.7-3.3 MHz). In real-time dynamic three-dimensional pattern, the frame rates of echocardiographic image were 25-50 frames/s. When the entire LV myocardium, endocardium, and epicardium were clearly displayed in apical 4-chamber view, we told the patients to hold their breath after a calm breath in order to eliminate breathing motion artifacts. Furthermore, to make sure a clear image, it is necessary to adjust the buttons that control the depth and width of the probe during the operation. Then turning into $4 \mathrm{D}$ full volume pattern, we continued to collect real-time dynamic three-dimensional images of LV in four consecutive cardiac cycles which can be analyzed with 3D-STE. At last, starting 4-dimensional automatic LV quantitative analysis software (4D-Auto LVQ) to analyze all the collected full-volume images. This software will automatically delineate the edocardial boundary of the LV, which can be manually adjusted if required. The parameters including LV end-diastolic volume (LVEDV), LV end-systolic volume (LVESV), LV ejection fraction (LVEF) were measured and strain curves for all 17 segments of the LV wall were obtained. Global longitudinal strain (GLS), global area strain (GAS), global radial strain (GRS) and global circumferential strain (GCS) of 17 myocardial segments in each direction were got by computing the weighted average of the peak systolic strain values. Each parameter was measured three times by a physician, and the average value was taken.

We analyzed all data using SPSS 19.0 software (SPSS 19.0, Inc., Chicago, IL, USA). All data conforming to normal distribution were showed as mean values+-standard deviations. Comparisons of the gender among the three groups were made by using $\chi^{2}$-test. Differences of more than two groups were compared by analysis of one-way ANOVA. And we compared the differences between two groups by using LSD-t test. To obtain the correlations between 3D-STE parameters and NT-proBNP in three groups, we used Pearson linear correlation analysis. The values of $\mathrm{P} 0.05$ demonstrated that the results were statistically significance. 


\section{Results}

\section{Clinical characteristics and NT-proBNP}

There was no obvious difference among the three groups of participants in the age, gender, BMI, BP and HR (all $\left.\mathrm{P}_{\dot{\iota}} 0.05\right)$. In terms of level of NT-proBNP, group $\mathrm{C}$ was the highest among three groups, and group $\mathrm{B}$ had higher NT-proBNP level than that in group A (all $\mathrm{P} ; 0.05)$.(Shown in Table 1)

\section{Conventional echocardiographic parameters}

No difference was showed in IVSTd and PWTd among three groups (both $\mathrm{P}_{\mathrm{j}} 0.05$ ). In group C, LAVImax , E/ mean e', LVDs and LVDd were increased (all $\mathrm{P} j 0.05$ ), while E/A, LVEF, Sept e' and Lat e' were decreased (all $\mathrm{P} ; 0.05$ ) compared to groups $\mathrm{A}$ and $\mathrm{B}$. In group $\mathrm{B}$, we found that LAVImax and $\mathrm{E} / \mathrm{mean}$ e' were increased, while Sept e' and Lat e' were decreased compared with group A (all $\mathrm{P}_{\mathfrak{1} 0.05}$ ). There were no obvious difference in terms of LVDs, LVDd, E/A and LVEF between groups A and B (all Pí0.05).(Shown in Table 2)

\section{D and 3D-STEparameters}

Compared with groups $\mathrm{B}$ and $\mathrm{A}$, LVEDV and LVESV were obviously increased (all $\mathrm{P} ; 0.05$ ), whereas LVEF

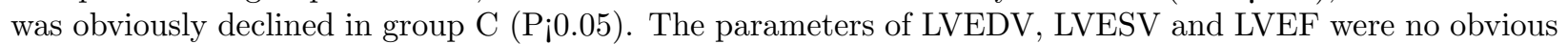
differences in groups $\mathrm{A}$ and $\mathrm{B}$ (all $\mathrm{P} \dot{0} 0.05$ ). GLS, GRS, GCS, GAS in group $\mathrm{C}$ were obviously lower (all $\mathrm{P}$ 0.05) than those in groups A and B. GLS, GCS and GAS in group B were lower than those in group A (all $\left.\mathrm{P}_{\mathfrak{i}} 0.05\right)$ and GRS was also reduced, but there was no difference $\left(\mathrm{P}_{\dot{\iota} 0.05)}\right.$. (Shown in Table 3 and Figure 1)

\section{Correlation analysis}

In order to make the results more intuitive, we used the absolute values instead of negative value of threedimensional strain parameters in the Pearson linear correlation. GLS, GRS, GCS and GAS were negatively related to NT-proBNP in group $\mathrm{C}(\mathrm{r}=-0.866 、 \mathrm{r}=-0.587 、 \mathrm{r}=-0.428 、 \mathrm{r}=-0.600 、 \mathrm{P}<0.001 、 \mathrm{P}=0.001 、 \mathrm{P}$ $=0.020 、 \mathrm{P}=0.010)$. (shown in Figure 2). And GLS is the only parameter that demonstrated a negative relationship with NT-proBNP in group B $(\mathrm{r}=-0.642 、 \mathrm{P}<0.010)$. (shown in Figure 3 )

\section{Discussion}

As a subtype of CHD, TVD is more serious than single-vessel and double-vessel disease, which has a higher incidence of cardiovascular events and mortality ${ }^{[10]}$. The LVEF is the most common parameter to evaluate myocardial systolic function ${ }^{[11]}$, but relevant studies have shown that the sensitivity of it is poor in detecting mild myocardial dysfunction which can be is detected quantitatively by measuring ventricular wall deformation ${ }^{[12]}$. Speckle tracking is a new echocardiographic technique developed in recent years. At present, 2D-STE has been widely used in clinical and experimental research ${ }^{[13,14]}$. But the limitation of 2D-STE is that the tracking of myocardial acoustic spots is limited to the two-dimensional section, which makes it untraceable when some of the spots move completely outside the two-dimensional section. The 3DSTE overcomes the'out-of-plane'phenomenon of $2 \mathrm{D}-\mathrm{STE}$ in scanning ${ }^{[15,16]}$. It can quantitatively evaluate the deformation of regional myocardium in all directions, and reflect motion and function of myocardium in three-dimensional space more realistically and accurately ${ }^{[17,18]}$. Crosby et al ${ }^{[19]}$ study on local myocardial function using speckle tracking technology shows that 3D-STE can accurately identify the myocardium with local myocardial dysfunction.

In our study, the LV structure of the group B was no obvious difference, and LVEF was also within the normal range compared with group A. However, the group C had larger LV volume and lower LVEF than those in other two groups. The left ventricular structure and LVEF can remain normal in group B, because the motion of myocardium in the ischemic region may be compensated by adjacent region myocardium. With the deterioration of disease, insufficiency of myocardial blood supply can cause ischemia, hypoxia and abnormal energy metabolism of myocardial cell, and even the loss of myocardial cell, and which further develops into diffuse myocardial fibrosis. Progressive aggravation of myocardial load gives rise to LV dilatation and 
increase of myocardial oxygen consumption which exacerbates the vicious cycle of myocardial overload and LV dilation, eventually leading to heart failure ${ }^{[20]}$. Kane et al ${ }^{[21]}$ have shown that the decrease of LV diastolic function is earlier than that of systolic function in patients with CHD. Our study evaluated LV diastolic function in line with the guideline issued by the American society of echocardiography in $2016^{[22]}$, which proved that the LV diastolic function was decreased in group B and group C. This is consistent with the results of Rydberg et al ${ }^{[23]}$, indicating that LV diastolic function is closely related to the degree of coronary artery stenosis. Due to TVD, the myocardial blood and energy supply is insufficient leading to the decrease of LV diastolic dysfunction, which is earlier than the change of systolic dysfunction in group B. With the aggravation of TVD, the degeneration and necrosis of the myocardium can result in fibroplasia and scar formation. Ultimately, myocardial compliance is reduced and ventricular diastolic function is impaired.

The myocardium of the LV is divided into three layers: the inner layer of the right hand spiral, the middle layer of the ring spiral and the outer layer of the left hand spiral ${ }^{[24,25]}$. Due to the orientations of myocardial fibers are different, its movement directions are also different. When the inner layer and outer layer myocardial fibers contract, there is movement in the direction of the long axis, and when the middle layer myocardium contracts, there is movement in the direction of the short axis. GLS, GCS and GRS respectively represent the myocardial deformation in the longitudinal, circumferential and perpendicular directions of endocardium. In addition, GAS is a new parameter ${ }^{[26,27]}$, which is the integration of GLS and GCS, and it represents the area change rate of endocardium. Longitudinal motion of myocardium is mainly determined by the myocardium fibers in the inner layer, and circumferential and radial motion are determined by the myocardium fibers in the middle layer ${ }^{[28,29]}$.

Our study found that the GLS in groups B and C were obviously diminished compared to control group, and the changes were statistically significant. Blood supply to the endocardium comes from the vessels at the end of the coronary artery and studies have shown that change of GLS is dominated by the endocardial myocardium ${ }^{[30]}$. So the endocardium is more sensitive to ischemia and hypoxia when the patients with TVD have myocardial ischemia, which means that GLS has changed under normal conditions of $\operatorname{LVEF}^{[31]}$. If stenosis rate of TVD further aggravates leading to the overload of cardiac and the enlargement of cardiac, the decrease of GLS is more significant. In our study, it was found that the GCS in groups B and C were obviously lower than that in control group $\left(\mathrm{P}_{j} 0.05\right)$. The pericardial contraction of the short axis of the cardiac is mainly caused by the movement of the middle layer, and the inner layer is also involved. Generally, myocardial ischemia gradually expands from the endocardium to the epicardium ${ }^{[32]}$, so when myocardial ischemia and hypoxia occur, the inner layer and the middle layer of myocardial segments are affected, resulting in reduction of GCS. GRS represents the thickness of the ventricular wall and the change of the LV volume from the LV short axis view ${ }^{[33]}$. In our study, the reduction of GRS in groups A and B were not statistically significant. It might be because the radial movement is closely related to the annular middle layer, and the had not completely involved the middle layer of the myocardium in this study. Furthermore, the radial thickening of ventricular wall is the result of the interaction of cross fibers between different myocardial layers and the inner and outer myocardium fibers perpendicular to fiber orientation. The strains of endocardial and epicardial myocardium fibers are similar in the orientation of parallel myocardial fibers, while it contributes less to GRS in the orientation of perpendicular to myocardial fibers. In the group C, GRS was obviously decreased compared with the other two groups. It showed that the further development of the disease had affected the middle layer, which was consistent with the research results of Winter et al ${ }^{[34]}$. As a specific indicator of 3D-STE, GAS is the integration of GLS of the ventricular long axis and GCS of the ventricular short axis, so the significant reduction of GAS can better assess the cardiac systolic dysfunction in patients with TVD.

NT-proBNP is produced by the hydrolysis of proBNP which can produce BNP at the time, and NT-proBNP may be more specific to cardiac activity than BNP ${ }^{[35]}$. Studies have confirmed BNP may be actively degraded in peripheral blood, so the test of NT-proBNP is more reliable ${ }^{[36]}$. NT-proBNP, as a polypeptide neurohormone, is mainly synthesized and secreted by ventricular myocytes ${ }^{[37]}$. In recent years, more and more attention has been paid to the diagnostic and prognostic of BNP in patients with different clinical types of $\mathrm{CHD}^{[38-40]}$. Some studies have showed the change of serum NT-proBNP closely related to myocar- 
dial ischemia and hypoxia. When the systolic function of cardiac is abnormal, the ventricular volume load increases and the ventricular wall is pulled, causing the synthesis and release of NT-proBNP ${ }^{[41]}$. Our results showed the level of NT-proBNP was distinctly difference among the three groups. NT-proBNP of group $\mathrm{C}$ was highest among of three groups. It was due to the severe stenosis of the TVD result in ischemia of cardiomyocytes and LV systolic dysfunction, which stimulates the synthesized and secreted of NT-proBNP by ventricular myocytes. In group C, the study demonstrated each 3D-STE strain parameter were negatively correlated with NT-proBNP. And the correlation analysis also demonstrated GLS was negatively correlated with NT-proBNP in group B. Therefore, the level of serum NT-proBNP is consistent with the 3D-STE in the evaluation of LV systolic function in patients with TVD.

\section{Study limitations}

The present study still existed some limitations. First of all, 3D-STE is not suitable for image acquisition of patients with irregular heart rhythm, and it requires the complete image information, which makes the loss of image information for patients with oversized heart. In addition, the high frame rate can accurately reflect the instantaneous motion information of the myocardium, while the low frame rate is adverse to real-time response of myocardial motion information. Furthermore, we had a relatively small study population, so it is necessary to expand the sample size for further study in the future.

\section{Conclusions}

All in all, LV function is closely related to the degree of coronary artery stenosis. If there is no timely intervention in the early stage of myocardial ischemia, the LV function will further deteriorate. As a new technique, 3D-STE can detect the changes of myocardial function earlier in patients with TVD without myocardial infarction, and quantitatively evaluate the global LV function through multiple global strain parameters. This may provide a more effective method for monitoring the change of LV systolic strain and evaluating LV systolic function of TVD without myocardial infarction.

\section{Contribution}

Greatly appreciated for the supports from Prof. Li, who obtained the 2D echocardiographic images, and participated in the analysis, interpretation and statistics of date with me, as well as critical revision. Xiuxiu Cui and Huaying Bo contributed equally to this article. Also thanks for the help of Dong Yu, MD, Ying Wang, MD, Ying Li, MD, Wenxing Chang, MD, Tingting, Yu, MD, approval of the article. Those authors are all from Department of Ultrasound, the Second Affiliated Hospital of Dalian Medical University, Dalian, China.

\section{Tables}

\section{Hosted file}

image1.wmf available at https://authorea.com/users/398665/articles/511300-three-dimensionalspeckle-tracking-echocardiography-in-evaluating-left-ventricular-function-in-patientswith-triple-vessel-coronary-artery-disease

Table 1: Clinical characteristics and NT-proBNP of the study population ()

\begin{tabular}{llllll}
\hline Parameters & Group A(n=30) & Group B $(\mathrm{n}=31)$ & Group B(n=31) & Group C $(\mathrm{n}=29)$ & Group C $(\mathrm{n}=29)$ \\
Age, years & $55.4 \pm 7.5$ & $57.4 \pm 8.3$ & $57.4 \pm 8.2$ & $57.4 \pm 8.2$ & $57.4 \pm 8.2$ \\
Systolic BP, mmHg & $115.3 \pm 8.03$ & $117.6 \pm 7.2$ & $121.3 \pm 9.0$ & $121.3 \pm 9.0$ & $121.3 \pm 9.0$ \\
Diastolic BP, mmHg & $78.0 \pm 3.2$ & $81.1 \pm 2.3$ & $82.4 \pm 3.2$ & $82.4 \pm 3.2$ & $82.4 \pm 3.2$ \\
HR, beats/min & $65 \pm 5$ & $65 \pm 4$ & $67 \pm 4$ & $67 \pm 4$ & $67 \pm 4$ \\
BMI, kg/m 2 & $24.5 \pm 2.5$ & $24.7 \pm 3.0$ & $25.3 \pm 3.3$ & $25.3 \pm 3.3$ & $25.3 \pm 3.3$ \\
NT-proBNP, pg/mL & $54.8 \pm 23.9$ & $394.3 \pm 147.3^{*}$ & $394.3 \pm 147.3^{*}$ & $394.3 \pm 147.3^{*}$ & $2476.6 \pm 1120.3^{* \#}$ \\
\hline
\end{tabular}


Abbreviations: $\mathrm{HR}=$ Heart rate; $\mathrm{BMI}=$ Body mass index; $\mathrm{BP}=$ Blood pressure

Note: *: pi0.05 versus group A. ${ }^{\#}: p_{i} 0.05$ versus group B

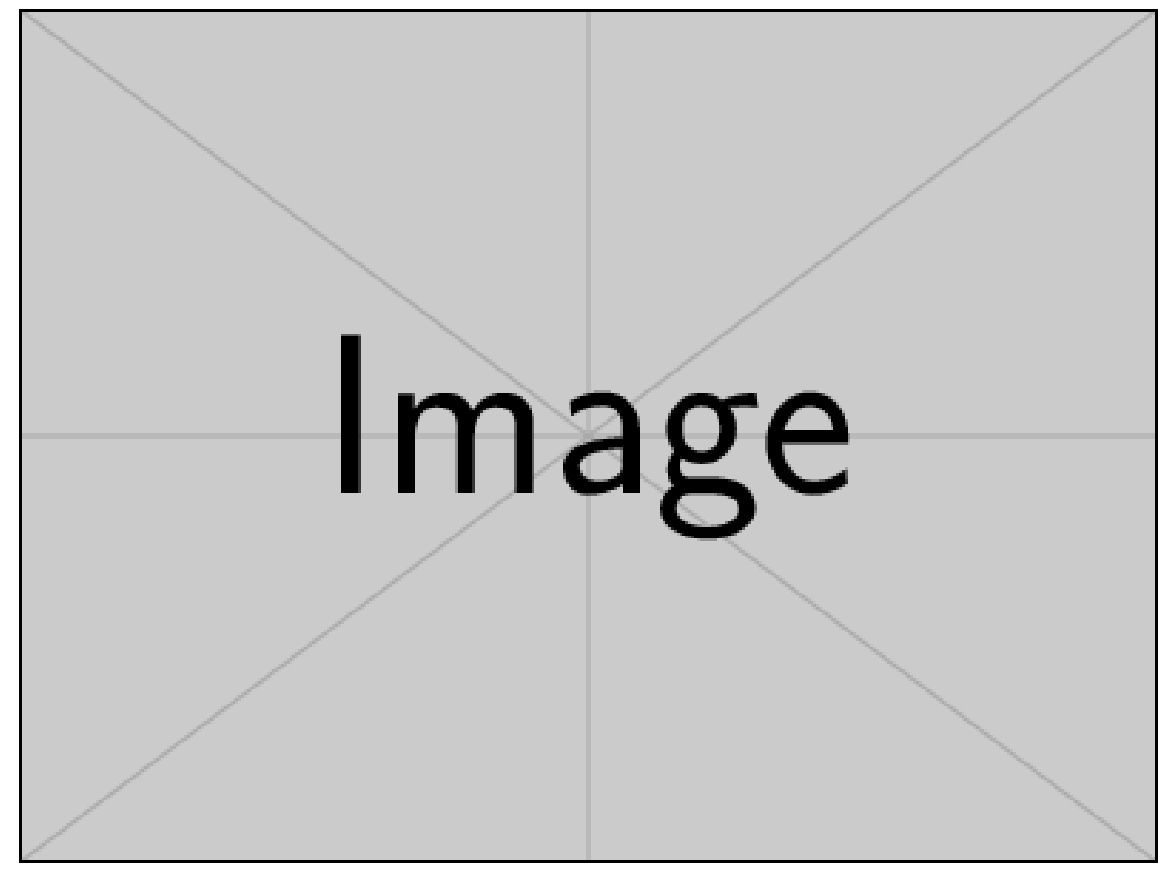

Table2: Conventional echocardiographic parameters of the study population ()

\begin{tabular}{lllllll}
\hline Parameters & Group A(n=30) & Group A(n=30) & Group B(n=31) & Group C(n=29) & Group C(n=29) \\
\hline LVDs ,mm & LVDs ,mm & $30.06 \pm 4.90$ & $31.13 \pm 4.76$ & $31.13 \pm 4.76$ & $48.01 \pm 6.09^{* \#}$ & $48.01 \pm$ \\
LVDd ,mm & LVDd ,mm & $46.08 \pm 3.74$ & $47.01 \pm 4.05$ & $47.01 \pm 4.05$ & $63.63 \pm 3.21^{* \#}$ & $63.63 \pm$ \\
IVSTd ,mm & IVSTd ,mm & $8.98 \pm 1.16$ & $9.10 \pm 0.68$ & $9.10 \pm 0.68$ & $9.37 \pm 0.68$ & $9.37 \pm 0$ \\
PWTd ,mm & PWTd ,mm & $9.12 \pm 1.00$ & $9.07 \pm 0.93$ & $9.07 \pm 0.93$ & $9.41 \pm 0.88$ & $9.41 \pm 0$ \\
E/A & E/A & $1.03 \pm 0.34$ & $0.95 \pm 0.22$ & $0.95 \pm 0.22$ & $0.73 \pm 0.41^{* \#}$ & $0.73 \pm 0$ \\
LAVImax ,mL/m $/ \mathrm{m}^{2}$ & LAVImax ,mL/m ${ }^{2}$ & $25.58 \pm 3.73$ & $37.29 \pm 5.44^{*}$ & $37.29 \pm 5.44^{*}$ & $43.27 \pm 6.88^{* \#}$ & $43.27 \pm$ \\
Sept e',cm/s & Sept e',cm/s & $10.22 \pm 2.87$ & $8.01 \pm 1.98^{*}$ & $8.01 \pm 1.98^{*}$ & $6.84 \pm 2.10^{* \#}$ & $6.84 \pm 2$ \\
Lat e',cm/s & Lat e',cm/s & $13.81 \pm 3.01$ & $10.41 \pm 2.74^{*}$ & $10.41 \pm 2.74^{*}$ & $9.44 \pm 2.36^{* \#}$ & $9.44 \pm 2$ \\
E/mean e' & E/mean e' & $7.61 \pm 2.02$ & $11.23 \pm 2.74^{*}$ & $11.23 \pm 2.74^{*}$ & $15.64 \pm 1.81^{* \#}$ & $15.64 \pm$ \\
\hline
\end{tabular}

Abbreviations: $\mathrm{LVDs}=\mathrm{LV}$ end-systolic diameter; $\mathrm{LVDd}=\mathrm{LV}$ end-diastolic diameter; IVST $=$ thickness of the interventricular septum; PWTd = end-diastolic thickness of left ventricular posterior wall; $\mathrm{E} / \mathrm{A}=$ ratio of peak early and late diastolic velocities; LAVImax = left atrial maximum volume index; Sept e'= septal mitral annular early-diastolic velocity; Lat e'=lateral mitral annular early-diastolic velocity; E/mean $\mathrm{e}^{\prime}=$ ratio of peak early diastolic velocities and septal and lateral early (e') mitral annular diastolic tissue velocities.

Note: *: p;0.05 versus group A. ${ }^{*}$ :p;0.05 versus group B 


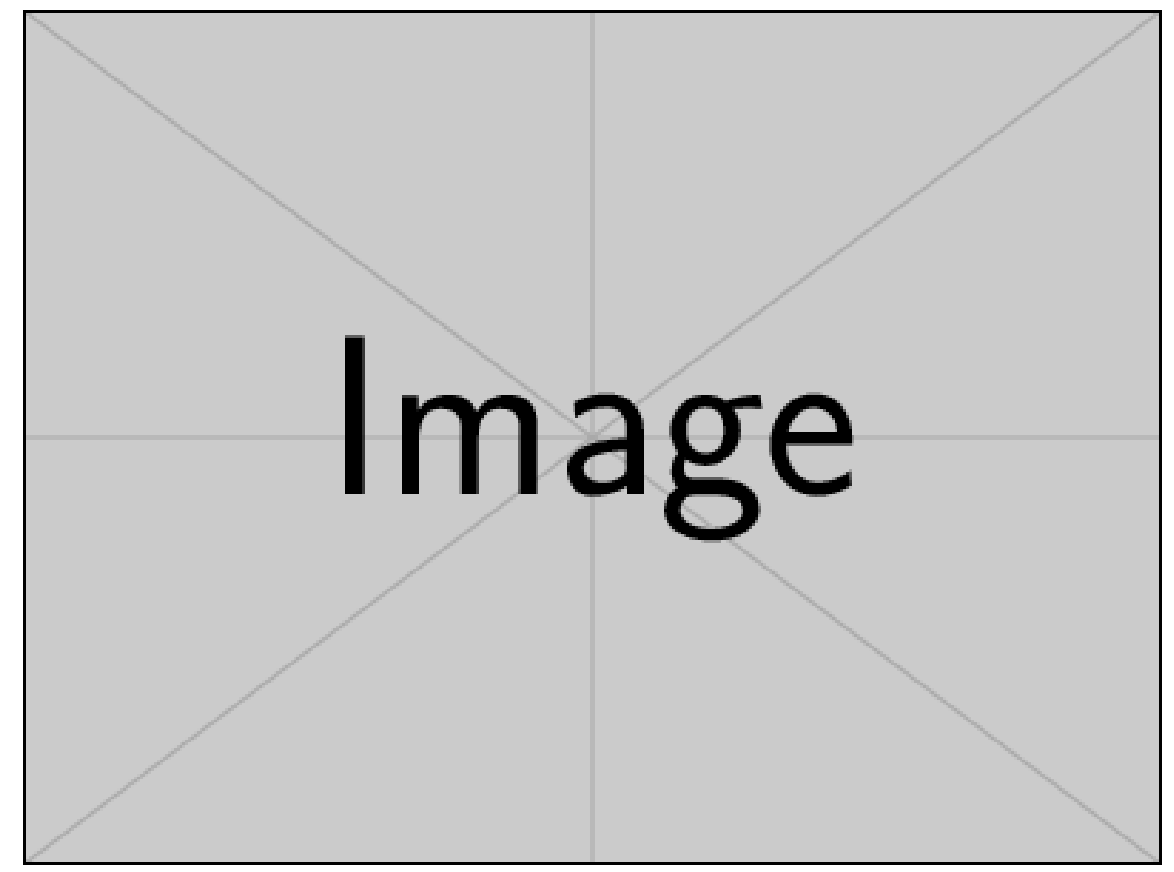

Table3: 3D parameters and 3D-STE parameters of the study population ()

\begin{tabular}{|c|c|c|c|c|c|}
\hline Parameters & Group $\mathrm{A}(\mathrm{n}=30)$ & Group $\mathrm{A}(\mathrm{n}=30)$ & Group $B(n=31)$ & Group B(n=31) & Group $\mathrm{C}(\mathrm{n}=29)$ \\
\hline LVEF , \% & $62.40 \pm 4.62$ & $60.50 \pm 3.91$ & $60.50 \pm 3.91$ & $35.5 \pm 5.43^{* \#}$ & $35.5 \pm 5.43^{* \#}$ \\
\hline $\mathrm{LVEDV}, \mathrm{mL}$ & $85.73 \pm 16.75$ & $87.35 \pm 15.80$ & $87.35 \pm 15.80$ & $201.62 \pm 22.86^{* \#}$ & $201.62 \pm 22.86^{* \#}$ \\
\hline LVESV ,mL & $32.30 \pm 9.11$ & $34.38 \pm 8.43$ & $34.38 \pm 8.43$ & $129.55 \pm 14.88^{* \#}$ & $129.55 \pm 14.88^{* \#}$ \\
\hline GLS,$-9.20 . \pm 2.35^{* \#}$ & $-9.20 . \pm 2.35$ *\# & & & & \\
\hline GRS, $19.27 \pm 2.60^{* \#}$ & $19.27 \pm 2.60^{* \#}$ & & & & \\
\hline GCS ,-8.93 $\pm 2.77^{* \#}$ & $-8.93 \pm 2.77^{* \#}$ & & & & \\
\hline GAS,$-15.41 \pm 4.35^{* \#}$ & $-15.41 \pm 4.35^{* \#}$ & & & & \\
\hline
\end{tabular}

Abbreviations: LVEF - left ventricular ejection fraction; LVEDV - left ventricular end-diastolic volume; LVESV - left ventricular end-systolic volume; GLS - global longitudinal strain; GRS - global radial strain; GCS - global circumferential strain; GAS - global area strain.

*: pi0.05 versus group A .\#:pi0.05 versus group B

\section{Reference}

1. Townsend N, Wilson L, Bhatnagar P, et al. Cardiovascular disease in Europe: epidemiological update 2016. Eur Heart J, 2016, 37: 3232-3245.

2. Benjamin EJ, Blaha MJ, Chiuve SE, et al. Heart Disease and Stroke Statistics-2017 Update: A Report From the American Heart Association. Circulation, 2017, 135: e146-e603.

3. Shimoni S, Gendelman G, Ayzenberg O, et al. Differential effects of coronary artery stenosis on myocardial function: the value of myocardial strain analysis for the detection of coronary artery disease. J Am Soc Echocardiogr, 2011, 24: 748-757.

4. Wang D, Zhang L, Zeng Q, et al. Assessment of left ventricular performance in heart transplant recipients by three-dimensional speckle tracking imaging. Medicine (Baltimore), 2017, 96: e8129. 
5. Nesser HJ, Mor-Avi V, Gorissen W, et al. Quantification of left ventricular volumes using threedimensional echocardiographic speckle tracking: comparison with MRI. Eur Heart J, 2009, 30: 1565-1573.

6. Zhou Q, Shen J, Liu Y, et al. Assessment of left ventricular systolic function in patients with iron deficiency anemia by three-dimensional speckle-tracking echocardiography. Anatol J Cardiol, 2017, 18: 194-199.

7. Voilliot D, Huttin O, Hammache N, et al. Impact of Global and Segmental Hypertrophy on TwoDimensional Strain Derived from Three-Dimensional Echocardiography in Hypertrophic Cardiomyopathy: Comparison with Healthy Subjects. J Am Soc Echocardiogr, 2015, 28: 1093-1102.

8. Li L, Zhang PY, Ran H, et al. Evaluation of left ventricular myocardial mechanics by three-dimensional speckle tracking echocardiography in the patients with different graded coronary artery stenosis. Int $\mathrm{J}$ Cardiovasc Imaging, 2017, 33: 1513-1520.

9. Montalescot G, Sechtem U, Achenbach S, et al. 2013 ESC guidelines on the management of stable coronary artery disease: the Task Force on the management of stable coronary artery disease of the European Society of Cardiology. Eur Heart J, 2013, 34: 2949-3003.

10. Ryu KS, Park HW, Park SH, et al. Comparison of clinical outcomes between culprit vessel only and multivessel percutaneous coronary intervention for ST-segment elevation myocardial infarction patients with multivessel coronary diseases. J Geriatr Cardiol, 2015, 12: 208-217.

11. Solomon SD, Anavekar N, Skali H, et al. Influence of ejection fraction on cardiovascular outcomes in a broad spectrum of heart failure patients. Circulation, 2005, 112: 3738-3744.

12. Yip GW, Zhang Q, Xie JM, et al. Resting global and regional left ventricular contractility in patients with heart failure and normal ejection fraction: insights from speckle-tracking echocardiography. Heart, 2011, 97: 287-294.

13. Langeland S, Wouters PF, Claus P, et al. Experimental assessment of a new research tool for the estimation of two-dimensional myocardial strain. Ultrasound Med Biol, 2006, 32: 1509-1513.

14. Leitman M, Lysyansky P, Sidenko S, et al. Two-dimensional strain-a novel software for real-time quantitative echocardiographic assessment of myocardial function. J Am Soc Echocardiogr, 2004, 17: 10211029 .

15. Biswas M, Sudhakar S, Nanda NC, et al. Two- and three-dimensional speckle tracking echocardiography: clinical applications and future directions. Echocardiography, 2013, 30: 88-105.

16. Thebault C, Donal E, Bernard A, et al. Real-time three-dimensional speckle tracking echocardiography: a novel technique to quantify global left ventricular mechanical dyssynchrony. Eur J Echocardiogr, 2011, 12: 26-32.

17. Hayat D, Kloeckner M, Nahum J, et al. Comparison of real-time three-dimensional speckle tracking to magnetic resonance imaging in patients with coronary heart disease. Am J Cardiol, 2012, 109: 180-186.

18. Tee M, Noble JA, Bluemke DA. Imaging techniques for cardiac strain and deformation: comparison of echocardiography, cardiac magnetic resonance and cardiac computed tomography. Expert Rev Cardiovasc Ther, 2013, 11: 221-231.

19. Crosby J, Amundsen BH, Hergum T, et al. 3-D speckle tracking for assessment of regional left ventricular function. Ultrasound Med Biol, 2009, 35: 458-471.

20. Folmes CD, Sowah D, Clanachan AS, et al. High rates of residual fatty acid oxidation during mild ischemia decrease cardiac work and efficiency. J Mol Cell Cardiol, 2009, 47: 142-148.

21. Kane GC, Karon BL, Mahoney DW, et al. Progression of left ventricular diastolic dysfunction and risk of heart failure. Jama, 2011, 306: 856-863. 
22. Nagueh SF, Smiseth OA, Appleton CP, et al. Recommendations for the Evaluation of Left Ventricular Diastolic Function by Echocardiography: An Update from the American Society of Echocardiography and the European Association of Cardiovascular Imaging. J Am Soc Echocardiogr, 2016, 29: 277-314.

23. Rydberg E, Willenheimer R, Erhardt L. The prevalence of impaired left ventricular diastolic filling is related to the extent of coronary atherosclerosis in patients with stable coronary artery disease. Coron Artery Dis, 2002, 13: 1-7.

24. Nakatani S. Left ventricular rotation and twist: why should we learn? J Cardiovasc Ultrasound, 2011, 19: $1-6$.

25. Goffinet C, Chenot F, Robert A, et al. Assessment of subendocardial vs. subepicardial left ventricular rotation and twist using two-dimensional speckle tracking echocardiography: comparison with tagged cardiac magnetic resonance. Eur Heart J, 2009, 30: 608-617.

26. Reant P, Barbot L, Touche C, et al. Evaluation of global left ventricular systolic function using threedimensional echocardiography speckle-tracking strain parameters. J Am Soc Echocardiogr, 2012, 25: 68-79.

27. Wang Q, Gao Y, Tan K, et al. Assessment of left ventricular function by three-dimensional speckletracking echocardiography in well-treated type 2 diabetes patients with or without hypertension. J Clin Ultrasound, 2015, 43: 502-511.

28. Chan J, Hanekom L, Wong C, et al. Differentiation of subendocardial and transmural infarction using two-dimensional strain rate imaging to assess short-axis and long-axis myocardial function. J Am Coll Cardiol, 2006, 48: 2026-2033.

29. Kim HK, Sohn DW, Lee SE, et al. Assessment of left ventricular rotation and torsion with twodimensional speckle tracking echocardiography. J Am Soc Echocardiogr, 2007, 20: 45-53.

30. Perk G, Tunick PA, Kronzon I. Non-Doppler two-dimensional strain imaging by echocardiography-from technical considerations to clinical applications. J Am Soc Echocardiogr, 2007, 20: 234-243.

31. Kraigher-Krainer E, Shah AM, Gupta DK, et al. Impaired systolic function by strain imaging in heart failure with preserved ejection fraction. J Am Coll Cardiol, 2014, 63: 447-456.

32. Mizuguchi Y, Oishi Y, Miyoshi H, et al. The functional role of longitudinal, circumferential, and radial myocardial deformation for regulating the early impairment of left ventricular contraction and relaxation in patients with cardiovascular risk factors: a study with two-dimensional strain imaging. J Am Soc Echocardiogr, 2008, 21: 1138-1144.

33. Nakai H, Takeuchi M, Nishikage T, et al. Effect of aging on twist-displacement loop by 2-dimensional speckle tracking imaging. J Am Soc Echocardiogr, 2006, 19: 880-885.

34. Winter R, Jussila R, Nowak J, et al. Speckle tracking echocardiography is a sensitive tool for the detection of myocardial ischemia: a pilot study from the catheterization laboratory during percutaneous coronary intervention. J Am Soc Echocardiogr, 2007, 20: 974-981.

35. Valli N, Gobinet A, Bordenave L. Review of 10 years of the clinical use of brain natriuretic peptide in cardiology. J Lab Clin Med, 1999, 134: 437-444.

36. Goetze JP, Jensen G, Moller S, et al. BNP and N-terminal proBNP are both extracted in the normal kidney. Eur J Clin Invest, 2006, 36: 8-15.

37. Hosoda K, Nakao K, Mukoyama M, et al. Expression of brain natriuretic peptide gene in human heart. Production in the ventricle. Hypertension, 1991, 17: 1152-1155.

38. Wei G, Yaqi R, Ningfu W, et al. N-terminal prohormone B-type natriuretic peptide and cardiovascular risk in stable coronary artery disease: a meta-analysis of nine prospective studies. Rev Cardiovasc Med, 2013, 14: e92-98. 
39. Eliasdottir SB, Klemenzson G, Torfason B, et al. Brain natriuretic peptide is a good predictor for outcome in cardiac surgery. Acta Anaesthesiol Scand, 2008, 52: 182-187.

40. Balta S, Demir M, Demirkol S, et al. Plasma B-type natriuretic peptide level in patients with coronary artery bypass surgery. Kaohsiung J Med Sci, 2013, 29: 648-649.

41. Chen TH, Lin CL, Shih JJ, et al. Plasma B-type natriuretic peptide in predicting outcomes of elective coronary artery bypass surgery. Kaohsiung J Med Sci, 2013, 29: 254-258. 

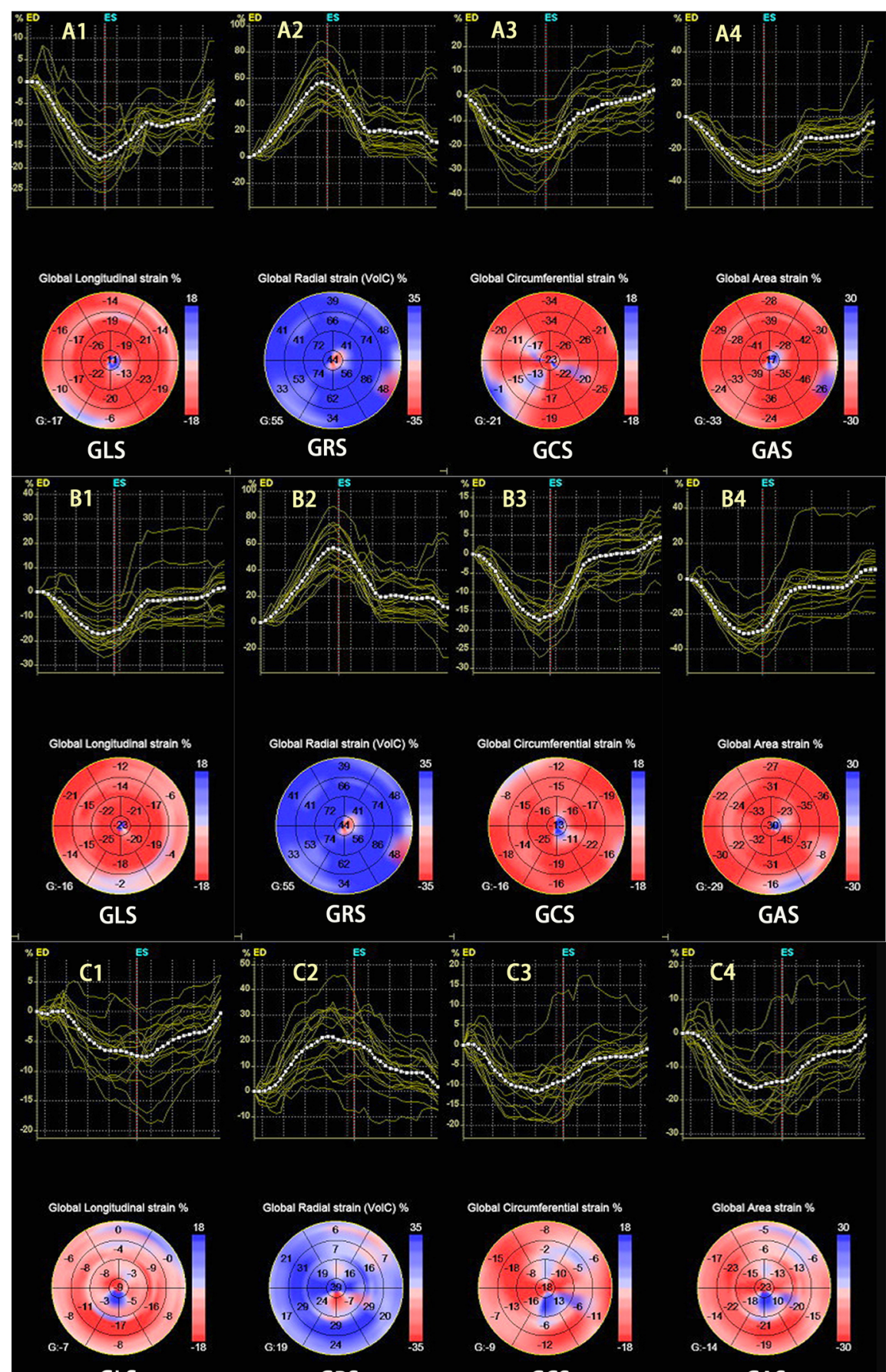

GRS
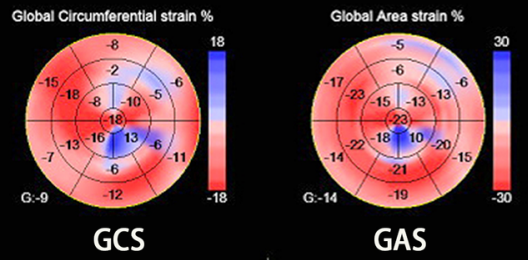

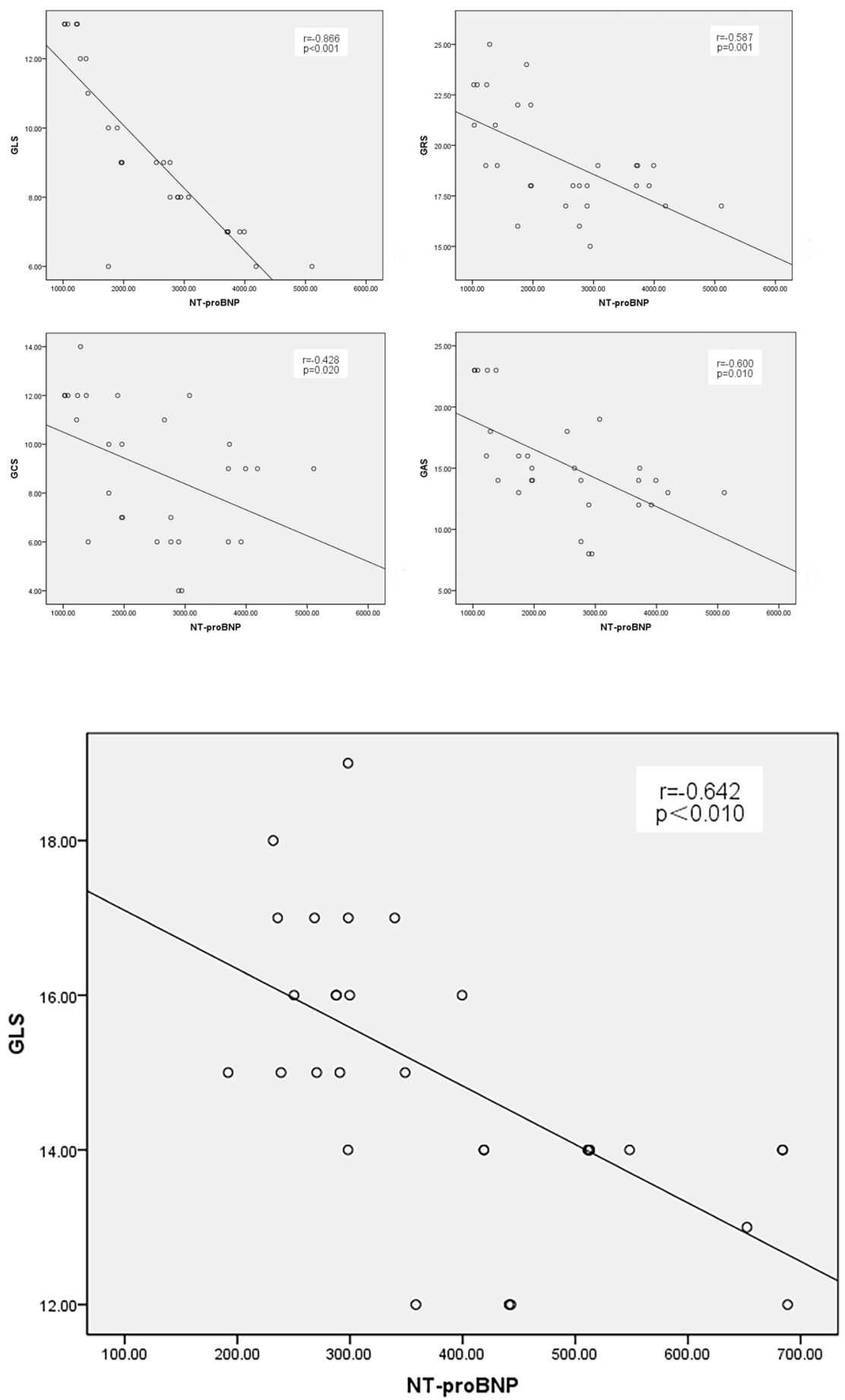